\title{
IDENTIFICATION OF THE ROOT CAUSE OF SURFACE TOPOGRAPHY INACCURACIES BY MEANS OF PROCESS MONITORING: INDUSTRIAL EXAMPLES
}

\author{
M. Gill ${ }^{1 *}$, X. Beudaert ${ }^{1}$, J. Munoa ${ }^{1,2}$, D. Barrenetexea1 , J.A. Sanchez ${ }^{2}$ \\ ${ }^{1}$ Ideko, Dynamics and Control, Elgoibar, Spain \\ ${ }^{2}$ University of the Basque Country, Department of Mechanical Engineering, Bilbao, Spain \\ ${ }^{*}$ Corresponding author; e-mail: mgil@ideko.es
}

\begin{abstract}
The surfaces of machined workpieces contain the signature of the manufacturing process. Indeed, the surface inaccuracies can be related to process parameters and machine vibrations. This paper aims at using process monitoring to identify the origin of surface topography defects. Thus, vibration and other process parameter measurements are correlated with profile measurements to establish the relationship between vibrations and surface topography for various process conditions. As each machine and process anomaly is reflected differently in the time and frequency domain, different analyses are applied both to temporal signals such as vibrations and to geometric signals obtained from the micro-geometry of the workpiece. Experimental results validate the diagnostic method and verify that the procedure is effective for the identification of topography defects originated not only from chatter vibrations but also from forced vibration coming from the kinematical chain (unbalance, beating...). The diagnostic method is successfully applied in various machining operations such as turning and grinding.
\end{abstract}

\section{Keywords:}

Chatter; Defects; Surface topography; Vibrations

\section{INTRODUCTION}

The quality of the finished product is measured in terms of its dimensional accuracy and surface integrity. Indeed, the surface topography is a significant factor in determining the performance of the workpiece and its importance depends upon its functional requirements. The classification, requirements and tasks involved in surface metrology are explained in the review of the measuring systems conducted in [Gao 2019].

The surface contains the footprint of the actual machining process [Whitehouse 1997]. Besides, the negative effect of vibrations on workpiece waviness has been demonstrated. Unbalance and wheel eccentricity [Badger 2011], chatter [Inasaki 2001] and beating phenomenon [Merino 2019] are some vibration sources that can create periodic marks on the workpiece surface. Defects on machine kinematic chain components such as bearings are also reported as the origin of surface topography marks [Bediaga 2013].

Lin and Chang [Lin 1998] developed a simulation model to predict surface finish considering the relative motion between the cutting tool and the workpiece in turning operations. Cao et al. [Cao 2013] presented a simulation model of surface topography for grinding processes considering both wheel surface topography and relative vibrations between grinding wheel and workpiece. The target was to optimize the grinding process and improve ground surface quality by considering process parameters such as the spindle speed, workpiece speed and depth of cut in the simulation model. Khare and Agarwal [Khare 2015] considered the random geometry and distribution of cutting edges on the wheel surface to develop a predictive model for surface roughness in grinding. Hockauf et al. [Hockauf 2019] used the actual grinding tool topography measured with a 3D laser triangularization sensor to predict the topography of ground surfaces. Kang et al. [Kang 2020] studied the influence of controlled vibrations onto simulated workpiece profiles in finishing turning operations.

Other authors have combined simulation models with actual process signals. Brecher et al. [Brecher 2019] presented a new approach where spindle integrated displacement sensors were employed to improve material removal simulations. They aimed to reproduce local effects of process forces and their influence on surface quality.

A review on the field of process monitoring regarding sensor technologies and signal processing techniques has also been published [Teti 2010]. Möhring et al. [Möhring 2010] integrated a sensory fixture that includes both sensors and a signal processing device to observe process and workpiece behaviour. In their work, Postel et al. [Postel 2019] estimated cutting forces and vibrations of a milling tool from accelerometers mounted at the spindle housing. 
Wavelet transform [Peng 2004], Fast Fourier Transform, Cepstrum analysis [Randall 2017] and Hilbert Transform [Yan 2006] are some of the post-processing methods that have been investigated for in-process measurements.

Although several authors have demonstrated that surface topography can be predicted using process monitoring and simulation models, little research has been done to relate the actual machine conditions to the surface topography. A further step includes correlating real process data and surface measurements to diagnostic machine defects.

Both surface topography measurements and process monitoring are key in the proposed methodology. Surface topography measurements determine if the ground part meets the quality requirements. Indeed, as each machine and process defect is reflected on the workpiece surface, the surface contains the footprint of the cutting process. The detailed analysis of the surface profile allows orienting the diagnostic in the adequate frequency band. Different process variables gathered from internal machine signals and physical sensors are analysed to monitor the cutting process. However, the relationship between process variables and their influence on the ground part is not straightforward. Thus, the correlation of actual process and surface topography signals enables an accurate diagnosis. The objective of this article is to present industrial applications of the combined analysis of surface topography and process monitoring. The paper serves as a guide to identify common industrial problems caused by surface topography inaccuracies.

\section{METHODOLOGY}

The methodology uses both surface topography and real process data to identify machine defects. The sequence of activities involved in machine tool diagnosis is gathered in Fig. 1. Once the workpiece is manufactured, machine state and process conditions are reflected on the final workpiece surface. The identification of any surface inaccuracy should initiate a review of the machine state to diagnose and correct the defect which is causing poor surface quality.

Surface topography measurements are carried out to analyse the surface profile. Clear periodic surface marks are directly related to vibrations frequency through the cutting speed (Eq. (1)).

$$
f=\frac{V_{c} \cdot 1000}{\lambda \cdot 60}
$$

Where $f(H z)$ is the marks generation frequency, $V_{c}(m / m i n)$ is the cutting speed and $\lambda(\mathrm{mm})$ is the marks wavelength.

In an established measuring distance, the number of marks is directly related to the wavelength by $E q(2)$.

$$
n=\frac{L}{\lambda}
$$

Where $\mathrm{n}$ is the number of marks, $\mathrm{L}(\mathrm{mm})$ the measuring distance and $\lambda(\mathrm{mm})$ the distance between marks. In turning operations, the measuring distance is the perimeter and, thus, the number of marks per revolution is quantified.

Errors like unbalance and misalignment provoke tool vibrations at a particular frequency. The regenerative effect of the cutting process can also self-excite any vibration mode of the machining system. Bearings, gears or belts failures are transmitted along the kinematical chain which causes periodic tool vibration. Besides, tool wear increases cutting forces and power consumption. Hence, vibrations, force, and power signals contain machine and process defects.

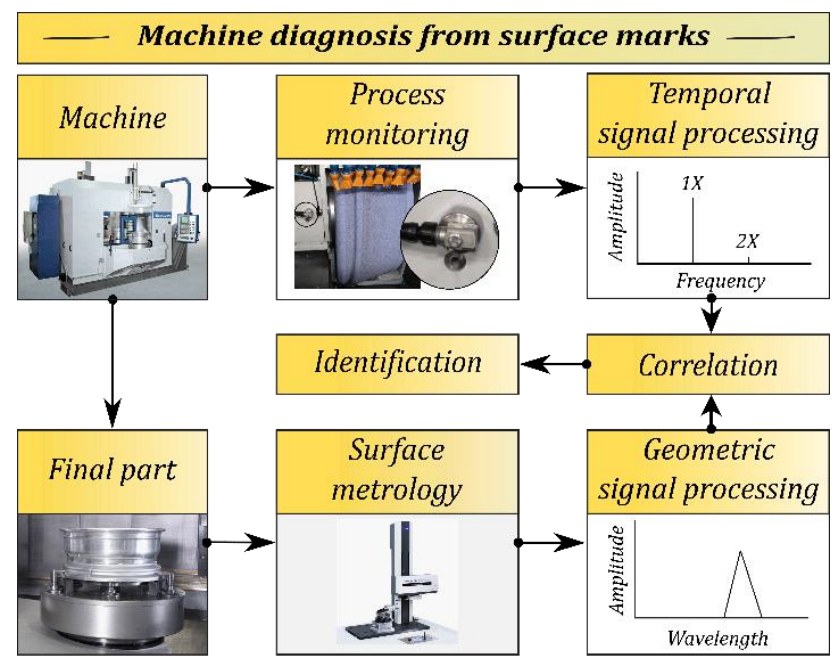

Fig. 1: Tasks for the identification of the origin of surface topography inaccuracies.

The first diagnosis of the vibrations' frequency narrows down the root cause of the defects which can be causing poor surface quality and the selection of the sensors to be used in process monitoring turns out to be more orientated to the faulty frequency. Process variables that can be related to machine defects and the material removal process are monitored with the appropriate physical sensors. Installing aspects and the measured frequency range are considered when choosing the appropriate sensors. Signals detected by power consumption, force and vibrations sensors are then processed to extract meaningful features from both the cutting process and the machine state.

Finally, the correlation of process monitoring and surface measurements will conclude with the identification and diagnosis of machine defects. The relation between the machine state and the final part might not be straightforward. Indeed, a particular frequency component of the vibration can be reflected as a complex surface profile. Besides, the influence of process forces on surface quality depends on tool geometry, process conditions and materials. Thus, a deeper analysis of the processes and physical phenomena that relate process variables and surface profile should be carried out.

\section{PROCESS MONITORING}

Typical machining process monitoring systems operate measuring process variables that reflect meaningful data regarding the cutting process. Process quantities such as cutting forces, vibrations and noise are widely used to monitored machining processes. Other process variables

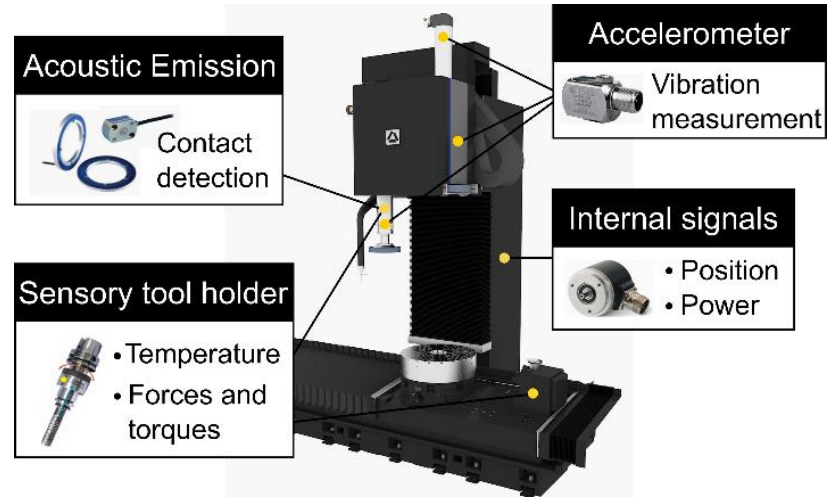

Fig. 2: Grinding machine's process monitoring capabilities. 
such as power consumption directly obtained from CNC are also a great source of information (see Fig. 2).

\subsection{Sensors}

Internal signals provided by electric drives and spindles such as motor power and motor current can be measured to monitor machine and drive states. This approach does not disturb the machining process. A major advantage of this measuring technique is its low cost. The sampling frequency is typically between $500 \mathrm{~Hz}$ and $2 \mathrm{kHz}$. However, motor and drive dynamics should be considered when processing the signals. Indeed, most of the encoders and linear measurement devices are far from the tool centre point and have limited bandwidth.

While motor power and current are implemented as an indirect measurement of the cutting forces, signals obtained from the encoders provide information related to the tool path and vibrations. Although indirect measurements are less accurate, their simplicity makes them more practical than direct techniques.

Accelerometers are usually the preferred additional sensors to monitor vibrations due to the ease of installation and low cost. The most common industrial accelerometers are based on the connection of a piezoelectric element to an inertial mass. Cost-effective industrial accelerometers permit the measurement until $5 \mathrm{kHz}$, an interesting frequency range for process and machine element condition monitoring. Most of the grinding machines have accelerometers on board for the automatic balancing systems. The bandwidth can be increased until $20 \mathrm{kHz}$. Notice that these sensors present limitations to measure low-frequency vibrations that can be overcome through piezoresistive accelerometers or velocimeters.

The location of the accelerometer has an important influence on the measurement quality. Practical issues such as the need for rotating axis or chip removal make it difficult to locate the sensor near the tool tip and low sensitivity to detect some grinding problems has been reported [Merino 2019]. Additionally, the vibration value depends on the receptance or dynamic flexibility of the machine. Therefore, in some frequencies the sensitivity can be low (antiresonances).

Acoustic pressure sensors such as microphones are employed to measure noise and vibrations. They are a good option for high-frequency vibrations related to local modes of parts and the tools. However, their signals are not completely reliable because they are capturing noises originated out of the machine.

The direct measurement of forces overcomes the drawback of vibration sensors due to the high sensitivity and rapid response to changes in the cutting state. Indeed, cutting forces reflect machine and process conditions such as tool failure. The main spindle torque provides a measurement directly related to the tangential force and different drives can help in the estimation of the cutting force. However, the low broadband of the internal signal and permutations coming from drive wear have driven researchers to include additional force sensors in the force flow of machine tools. Piezoelectric force cells and dynamometers can be used but the high price and their fragility have limited their applications in industrial use. The load cells based on strain gages are another alternative. Their sensitivity depends on the created stress concentration weakening a little bit the machine element. A good balance between the stiffness of the element and the sensitivity is key to the application.

Recently, tool holders with strain measurement capabilities (Fig. 3) have been launched [Bergs 2019]. These tool holders permit to have an indirect estimation of the radial (a)

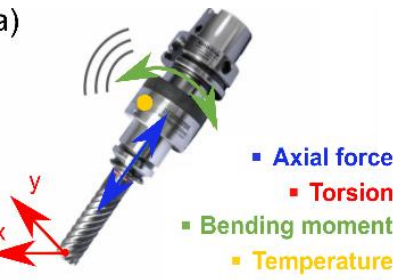

(b)

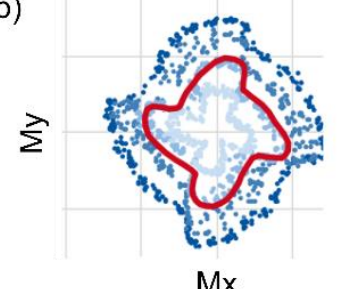

$\mathrm{Mx}$

Fig. 3: Sensory tool holder from Pro-micron: (a) Measuring capabilities; (b) Bending moments' polar plot [Bergs 2019].

forces that are more sensitive to process variations including tool wear.

Finally, Acoustic Emission ( $A E$ ) sensors can be used to monitor the cutting process [Ravindra 1997]. These sensors are really sensitive to detect machining anomalies, but present a lack of robustness for industrial applications. However, they are successfully used to predict the contact between the wheel and workpiece in grinding machines.

\subsection{Signal processing}

The combination of all the processes which take place during machining operations creates a complex signal that is processed to have a better view of the condition of the machine.

Time domain analysis can be carried out to extract single features such as average value, magnitude or root mean square value (RMS). Indeed, some standards classify the status of different machine elements based on these values (ISO 10816). However, deeper comprehension of periodic signals requires further analysis in the frequency domain to identify different frequency patterns. Applying Fast Fourier Transform (FFT) algorithm, peaks at dominant frequencies of periodic signals are visualized. To guarantee accurate results, aspects such as signal sampling, ski slope, aliasing and leakage should be considered when defining the data acquisition parameters. FFT assumes the periodic and stationary character of the signal. However, when the input is not periodic in the time recording the FFT is computed based on a distorted signal. To minimize this effect the signals can be filtered by a Hanning window. The FFT doesn't permit to be accurate in frequency and time domain at the same time, and therefore it has limitations to deal with non-stationary signals.

A further interpretation of sideband families and determination of the frequency spacing among peaks can be conducted with Cepstrum analysis. The Cepstrum analysis calculates the spectrum of the logarithm of the energy spectrum. This technique discovers periodicities in the frequency spectrum. However, inaccuracies in the spacing of frequencies decrease the effectiveness of this method.

Amplitude demodulation using the Hilbert Transform can also be implemented to capture transient events in dynamic systems. An advantage of this algorithm is that it is not constrained by the limitations concerning the time and frequency resolutions.

A time-frequency representation of the signals is presented by Wavelet Transform. This technique enables higher signal-to-noise ratios and, thus, more accurate signal analysis [Wu 1996]. Indeed, Wavelet transform is useful to detect single events in transient signals such as the beginning of chatter vibrations or the instance of an impact [Stepan 2011].

The time-domain signals can be transformed to polar plots based on the values of two sensors measured in two perpendicular directions. Polar plots are especially useful for displacement sensors, permit to describe orbits of 
spindle and hydrodynamic bearings and allow to identify frictions and impacts. Polar plots can be used with force signals too, and tool wear and engagement can be identified [Brecher 2019]. With polar plots, the intersection of the force or moment measurements in two different directions is depicted. In such a way, the magnitude of the drawing determines the amount of force at work on the cutter at that given point and time and, thus, the force distribution along the cutting edges.

Each machine defect is reflected differently in the process dynamics. Hence, it is relevant to decide which process variables must be monitored. Besides, the signal's meaningful features must be identified to apply the appropriate post-processing technique. Indeed, while vibrations frequency components are observed at the frequency spectrum of acceleration and noise signals, polar plots reflect force distribution on the cutting edges.

\section{PART TOPOGRAPHY MEASUREMENT}

Workpiece quality requirements are defined in terms of dimensional accuracy and surface topography. Indeed, workpiece specifications are defined by form, waviness and roughness parameters. According to the field of application different sensing technologies and surface characterization methods have been developed.

\subsection{Sensors and methods}

A first visual inspection of the manufactured workpiece can be conducted. This is a fast and easy method to implement when the generated surface is made up of a unique frequency. The distance between marks is measured with a gauge or deduced from the number of marks left at a given measuring distance (Eq. (2)). Then, marks' wavelength is directly related to the fault frequency (Eq (1)). However, the low accuracy of the wavelength measurement introduces high incertitude.

Contacting sensors such as profilometers have been widely used to conduct topography measurements. The stylus method essentially uses a caliper with two arms that touch a reference surface and the surface under test. With this technology position or dimension is not measured. Typical stylus solutions enable measurements at $20 \mathrm{~Hz}$, a range of $50 \mu \mathrm{m}$ and a resolution of $0.3 \mathrm{~nm}$. Hand-held instruments such as portable profilometers are a solution for onmachine measurements. A stroke of $16 \mathrm{~mm}$ and ranges of $360 \mu \mathrm{m}$ with a resolution of $0.02 \mu \mathrm{m}$ are typical.

Coordinate measuring machines (CMM) can measure the surface topography using scanning touch probes. Although large size pieces can be measured, high measuring times are required for off-line measurements.

Non-optical methods such as capacitance sensors are another alternative for surface topography measurements. Some of these use an electrode positioned above the surface. The capacitance between the sensor and the surface is an indicator of the roughness. As the relationship between the capacitance value and the surface roughness follows an inverse law with nonlinear terms some complications may arise when using this technology. The attractive features rely on its high sensitivity, the implementation of a non-contacting method and the integration of the surface roughness by measuring over an area. Industrial capacitive sensors provide a bandwidth of $15 \mathrm{kHz}$, a range of $250 \mu \mathrm{m}$ and a peak-to-peak resolution of $34 \mathrm{~nm}$. Inductive displacement sensors with a range up to $1 \mathrm{~mm}$ overcome the low measuring ranges of capacitive sensors.
Ultrasounds have also potential use for surface measurements. In this technology, the scattered waves are picked up from the ultrasonic wave which is projected onto the surface. In addition to the amplitude, phase information can be obtained. However, very high frequencies are required.

The scanning technology is employed in optical sensors where light is projected onto the surface. The flying-spot microscope is a technology in which the illumination of the surface is restricted to a very small spot. The reflected light is picked up by a point detector which acts as a pinhole and blocks out all light from an out-of-focus plane. A variation of this technology is the confocal microscope in which the imaging and receiving optics are the same. The axial movement needed to keep the spot focus is related to the absolute roughness value. Resolutions of $0.1 \mu \mathrm{m}$ in depth are possible.

In-process measurement devices are typically used to measure the dimensional accuracy of the parts but the surface topography is generally not measured in the machine. First implementations of in-process microgeometry measurements have been implemented for grinding applications with continuous measurement of the surface topography of all parts exiting the machine [Kapłonek 2015]. This promising technology (Fig. 4) is based on the visualization of the scattered light which is picked up by two detectors. The surface roughness can be estimated from the ratio of the scattered light which hits the two detectors. Scattering light provides a robust, fast, and traceable measurement [Sommer 2019]. However, constraints arise due to the dependency of the lightscattering characteristics on the manufacturing process. With this method, a resolution of $1 \mathrm{~nm}$ and a range of 100 $\mathrm{nm}$ are obtained. Furthermore, the interferometer utilizes the coherent property of the light. In this case, the technology benefits from the interference between the light scattered from the test surface and the one from a reference surface. Resolutions of $1 \mathrm{~nm}$ and ranges of $1 \mu \mathrm{m}$ are usual.
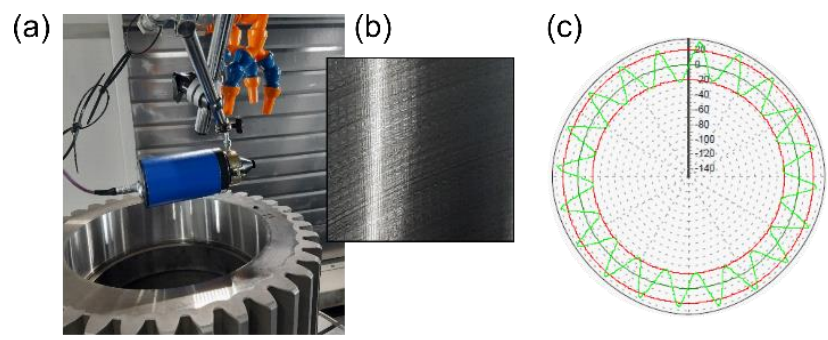

Fig. 4: Topography measurements with Optosurf technology: (a) Setup; (b) Visual lobes of the internal diameter of a wind gear; (c) Profile waviness signal.

\subsection{Signal processing}

Geometric parameters such as the average roughness ( $\mathrm{Ra})$ and the average peak-valley heigh $(\mathrm{Rz})$ are widely used to define the workpiece's quality requirements (ISO 4287 and ISO 4288). The Ra parameter gives an adequate statistical parameter that characterizes the amplitude distribution. However, it does not represent the irregularity form of the profile. Rz parameter is also adequate to characterize the surface of periodic profiles. However, it fails to define profile form, neither the distance between marks.

When analysing periodic profiles composed of a unique frequency the recount of the marks can be satisfactory. However, when the continuous marks are composed of several frequencies a further analysis is required.

For a clear distinction between roughness, waviness and form the measured surface needs to be filtered (ISO 
11562). Further, frequency spectrum analysis is useful to extract relevant features of the periodic processes. To this effect, the Fast Fourier Transform shows peaks at marks wavelength.

\section{EXPERIMENTAL PROCEDURE}

Three case studies where the manufactured workpiece reports poor surface quality are presented. To identify the root cause an analysis of the machine and process variables is carried out through process monitoring.

\subsection{Unbalance marks in grinding}

The identification of the origin of surface topography inaccuracies during a cylindrical plunge grinding operation is carried out. Machine components and process conditions are presented in Tab. 1 and Fig. 5a.

Tab. 1: System components of plunge cylindrical grinding.

\begin{tabular}{ll}
\hline $\begin{array}{l}\text { Grinding } \\
\text { wheel }\end{array}$ & SU33A 602 II10 VB1 from TYROLIT \\
$\begin{array}{l}\text { Dressing } \\
\text { disk }\end{array}$ & $\begin{array}{l}\text { HDPH 50AP from TYROLIT B185; } \\
\text { effective width 0,7mm }\end{array}$
\end{tabular}

(a)
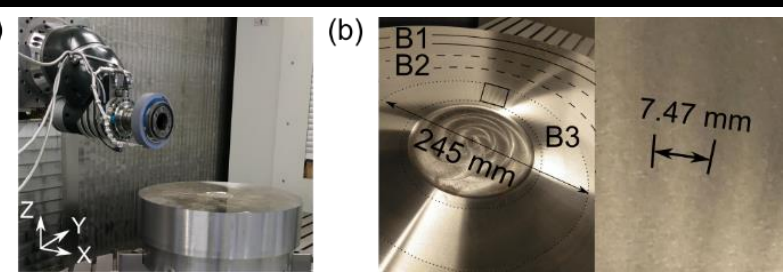

Fig. 5: Plunge cylindrical grinding: (a) Overview; (b) Unbalance surface marks.

Despite the good level of balance carried out and the low level of vibrations during grinding, the inaccuracies are visually evident (Fig. 5b). To measure surface topography a device which allows measurements on the machine and at a concrete diameter along $360^{\circ}$ is needed. As a portable profilometer has a limited stroke a capacitance sensor is used to measure the surface topography directly in the machine tool (Fig. 7). The waviness profile measured at a diameter of $195 \mathrm{~mm}$ is presented in Fig. 6.

The frequency spectrum of the profile shows clear peaks at a distance of $7.47 \mathrm{~mm}$, which is associated with a frequency of $34.09 \mathrm{~Hz}$ at a workpiece speed of $24.94 \mathrm{rpm}$ (Eq. (1)).

(a)

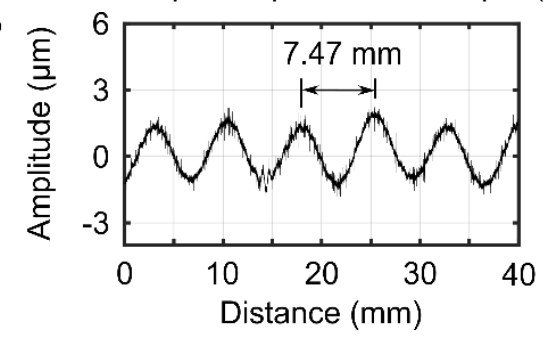

(b)

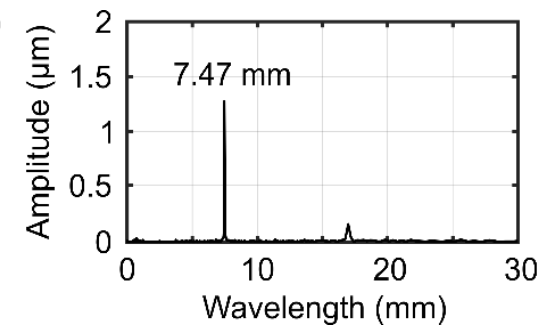

Fig. 6: Profile measurement of a ground part with unbalance marks; (a) Geometric signal; (b) Spectrum.
Tab. 2: Unbalance test's process conditions.

\begin{tabular}{ccccc}
$\begin{array}{c}\text { Grinding } \\
\text { wheel } \\
\text { speed } \\
\left(\mathrm{N}_{\mathbf{s}}\right)\end{array}$ & $\begin{array}{c}\text { Workpiece } \\
\text { speed }\left(\mathrm{N}_{\mathrm{p}}\right)\end{array}$ & $\begin{array}{c}\text { Feed } \\
\text { rate } \\
\left(\mathbf{V}_{\mathrm{f}}\right)\end{array}$ & $\begin{array}{c}\text { Measured } \\
\text { marks on the } \\
\text { workpiece }\end{array}$ \\
\hline $\mathbf{r p m}$ & $\mathbf{r p m}$ & $\mathbf{m m} / \mathbf{m i n}$ & $\mathbf{m m}$ & $\mathbf{H z}$ \\
\hline 2045 & 24.94 & 0.06 & 7.47 & 34.09 \\
\hline & & &
\end{tabular}

Fig. 7: Vibrations and surface topography sensors.

As low-frequency components are only detected by accelerometers, to monitor the grinding operation (Tab. 2) an accelerometer is located on the spindle head. Cutting forces monitoring is another alternative that allows estimating tool's displacement. However, the unavailability of a sensory tool holder that suits the tool makes this alternative unfeasible.

The frequency spectrum of the vibration's signals (Fig. 8) shows a clear peak at the grinding rotational speed (34.08 $\mathrm{Hz}$ ). Another main peak at $2 \mathrm{X}$ harmonic of the grinding wheel rotational speed is also reflected. The vibrations signals are observed at the frequency spectrum due to the periodic character of the unbalance of the grinding wheel The defect which is causing the waviness profile is identified, so no additional signal analysis is required.

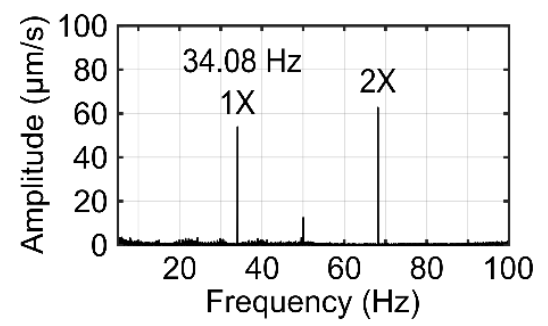

Fig. 8: Vibration spectrum during cylindrical grinding.

The waviness profile in the part is generated due to the unbalance of the first harmonic of the grinding wheel rotational speed. Unbalance or run out are reported as the main causes of this phenomenon. Thanks to surface topography measurements and the characterization of the profiles' wavelength this diagnosis procedure is bounded and adequate sensors to identify low-frequency components of the vibration's signals are employed.

\subsection{Chatter marks in turning}

High vibrations are reported when turning a workpiece with new inserts (Tab. 3). These vibrations are reflected as a poor visual aspect of the manufactured workpiece surfaces. The surface shows 134 marks per revolution with a steady

Tab. 3: Process conditions of a turning operation.

\begin{tabular}{ccccc}
\hline $\begin{array}{c}\text { Workpiece } \\
\text { inner } \\
\text { diameter }\end{array}$ & $\begin{array}{c}\text { Cutting } \\
\text { speed }\end{array}$ & $\begin{array}{c}\text { Feed } \\
\text { rate }\end{array}$ & \multicolumn{2}{c}{$\begin{array}{c}\text { Measured marks } \\
\text { on the workpiece }\end{array}$} \\
\hline $\mathbf{m m}$ & $\mathbf{m} / \mathbf{m i n}$ & $\mathbf{m m} / \mathbf{r e v}$ & $\mathbf{m m}$ & $\mathbf{H z}$ \\
\hline 32 & 150 & 0.2 & 0.82 & 3048.78 \\
\hline
\end{tabular}


(a)

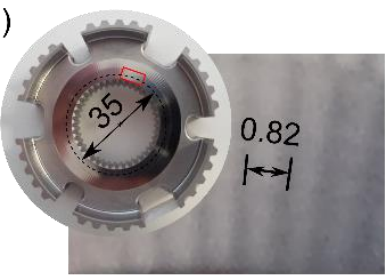

(b)

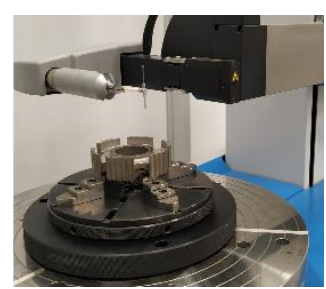

Fig. 9: (a) Turning chatter surface marks; (b) Profile measurements with a contacting profilometer.

distance of $0.82 \mathrm{~mm}$ at a diameter of $35 \mathrm{~mm}$ (Fig. 9a). This wavelength corresponds to a generation frequency of $3048 \mathrm{~Hz}$ at a cutting speed of $150 \mathrm{~m} / \mathrm{min}$ (Eq. (1)).

Profile measurements conducted by a profilometer at a diameter of $35 \mathrm{~mm}$ (Fig. 9b and Fig. 10) corroborate the $0.82 \mathrm{~mm}$ wavelength. A second frequency component at $18.33 \mathrm{~mm}$ is reflected in the spectrum. This value corresponds to the waviness shape caused by the 6 prominences of the workpiece which are uniformly distributed along the perimeter.

(a)

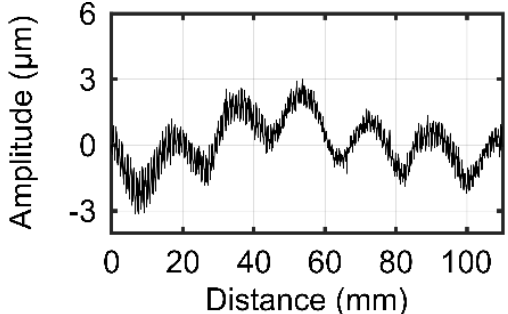

(b)

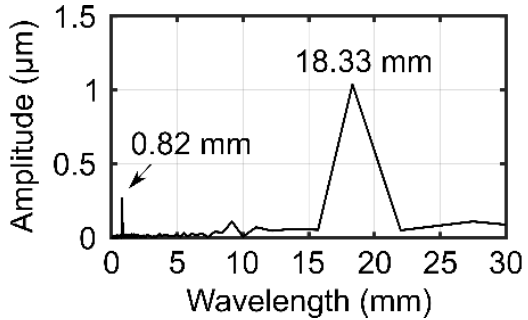

Fig. 10: Profile measurement of a turning operation with chatter marks; (a) Geometric signal; (b) Spectrum.

To identify the machine defect, the adequate sensors for high-frequency components' identification are selected. As the turning operation of the workpiece requires its location at the tool holder, the use of a sensory tool holder is discarded. Internal signals' range is lower than the frequency observed at the surface profile $(3048 \mathrm{~Hz})$.

(a)

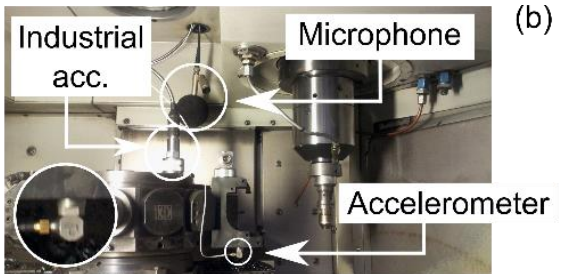

Fig. 11: Vibration sensors for a turning operation.

(a)

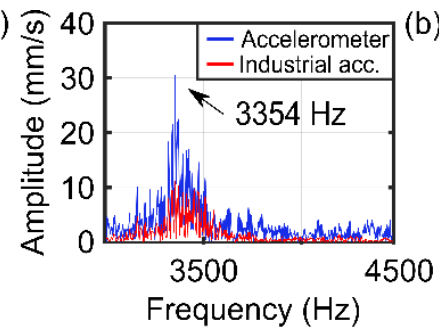

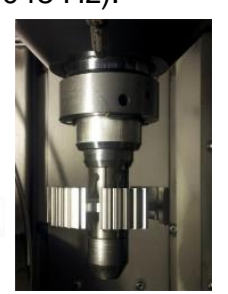

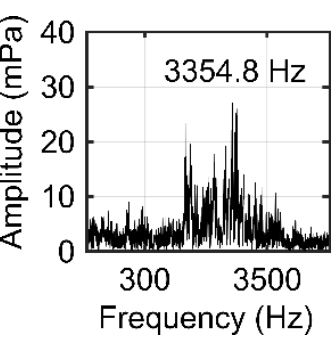

Fig. 12: (a) Acceleration's and (b) microphone's frequency spectrum for turning.

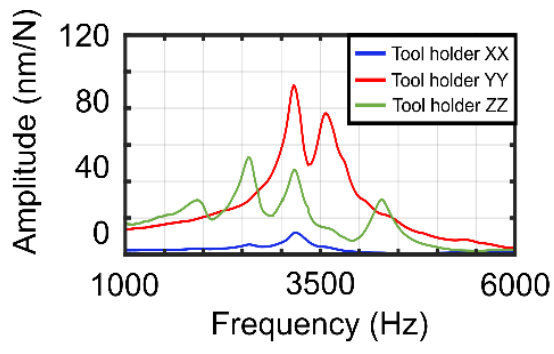

Fig. 13: Tool holder Frequency Response Functions (FRF).

To identify components around $3 \mathrm{kHz}$, two accelerometers are mounted on the tool and turret (Fig. 11). Additionally, a microphone is used to monitor noise levels during the process. Noise sensors are suitable for high-frequency components identification. The frequency spectrum of the collected signals (Fig. 12) shows a dominant peak at a frequency of $3354 \mathrm{~Hz}$.

Dynamic analysis of the tool (Fig. 13) corroborates that the regenerative effect is originated due to the excitation of a tool mode. The modal analysis (Fig. 14) shows that the insert holder vibrates with a bending mode in both tangential and axial directions. (a)

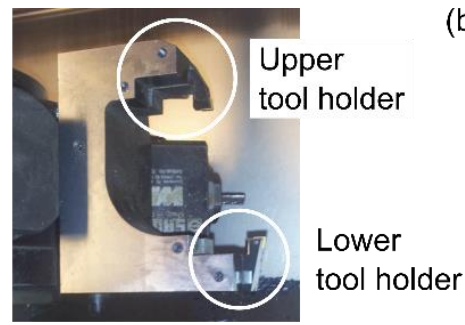

(b)

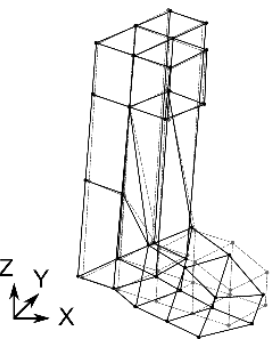

Fig. 14: (a) Tool holder geometry; (b) Tangential direction ( $Y$ ) tool holder bending mode at $3164 \mathrm{~Hz}$.

The frequency component at $3354 \mathrm{~Hz}$ is caused by the regenerative effect of the cutting process. When the tool wear increases, this phenomenon disappears. This accounts for the so-called process damping. Inserts wear increases the contact zone between the tool and the workpiece. Consequently, the friction between the insert and the workpiece increases generating additional damping which stabilizes the process.

Thanks to both surface topography and process measurements the diagnosis has been made. Surface topography's low wavelength has been related to highfrequency vibrations. Thereby, process monitoring has been narrowed down to identify high-frequency components which are well detected by accelerometers and acoustic pressure sensors

\subsection{Beating marks in cylindrical grinding}

The analysis of the waviness profile of a ground rotating slender workpiece is performed (Tab. 4; Fig. 15). Several tests ( $T a b .5)$ have been conducted to analyse the influence of process parameters on the surface finish.

Tab. 4: System components of cylindrical grinding.

\begin{tabular}{ll}
\hline $\begin{array}{l}\text { Grinding } \\
\text { wheel }\end{array}$ & Corundum CS65A 30,2 HH5 VB3 (C41) \\
Dressing & Infiltered disk \\
disk & 34368157 RIG 52035 3S1M R0,5 (D17) \\
& $\varnothing 120 \mathrm{~mm}$ \\
Workpiece & $\varnothing 50 \times 300 \mathrm{~mm}$
\end{tabular}




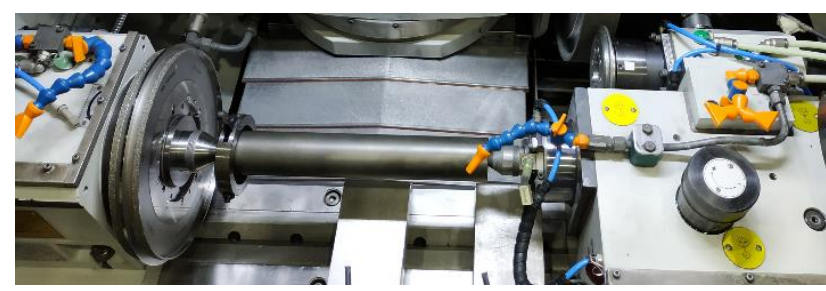

Fig. 15: Grinding of a slender workpiece.

(a)
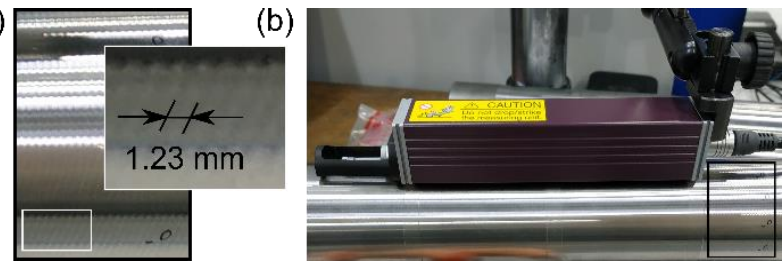

Fig. 16: Beating surface marks of cylindrical grinding; (a) Visual inspection; (b) Profilometer measurement.

After performing test A1 poor surface quality is observed. Indeed, vibrations leave a helix pattern on the workpiece surface. The measured axial distance between the periodic marks (Fig. 16a) is $1.23 \mathrm{~mm}$. The geometric signal and frequency components of the topography measurements carried out with a portable profilometer (Fig. 16b) are represented in Fig. 17. Ra parameter according to ISO 4288-1997 standards is presented in Tab. 5. A deeper analysis of the helix pattern can be conducted thanks to a 3D surface topography measurement with Optosurf technology [Sommer 2019].

A dominant wavelength of $1.23 \mathrm{~mm}$, which corresponds to a frequency of $2.71 \mathrm{~Hz}$ (Eq. (1)) at the given feed rate (200 $\mathrm{mm} / \mathrm{min}$ ) is observed. Other frequency components with a wavelength of $0.65 \mathrm{~mm}$ and $0.09 \mathrm{~mm}$ are also present in the spectrum.

(a)

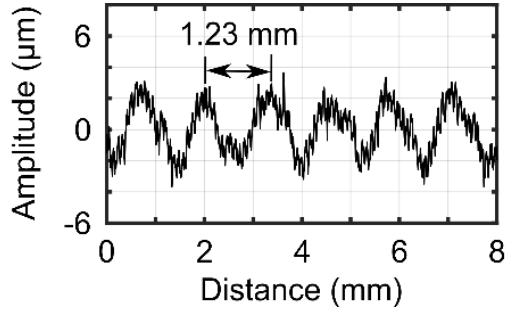

(b)

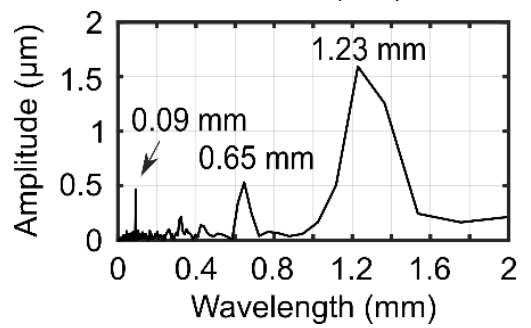

Fig. 17: Profile measurement of a cylindrical grinding with beating marks; (a) Geometric signal; (b) Spectrum. (a)
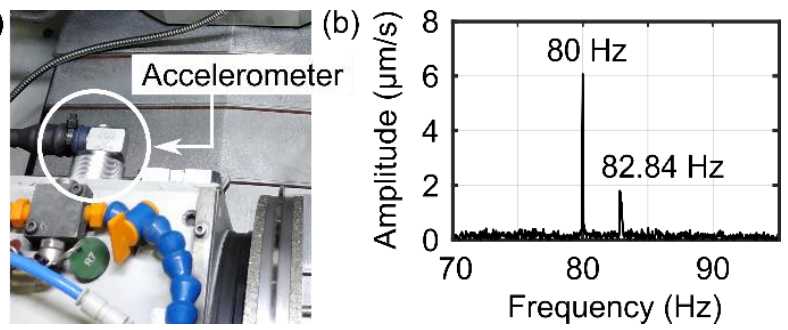

Fig. 18: Vibration's measurement during cylindrical grinding: (a) Accelerometer location at the workpiece head; (b) Frequency spectrum.

The profile wavelength corresponds to a very low-frequency which can be related to the beating phenomenon. For a deeper comprehension of the cutting process, process monitoring is carried out by accelerometers located in the workpiece spindle head (Fig. 18a).

The vibration signal collected during test $\mathrm{A} 1$ (Fig. 18b) shows two nearby peaks at $80 \mathrm{~Hz}$ and $82.84 \mathrm{~Hz}$, which corresponds to the $2 \mathrm{X}$ harmonic and $1 \mathrm{X}$ harmonic of the grinding wheel and dressing disk rotational speed respectively.

The frequency of the main wavelength (Fig. 17b) matches the difference between $2 X$ and $1 X$ harmonics of the grinding wheel and dressing disk rotational speeds. Another modulation of $4 X$ and $2 X$ harmonics of the grinding wheel and dressing disk rotational speeds is observed at a wavelength of $0.65 \mathrm{~mm}$. The dressing feed rate is reflected at the workpiece surface with a wavelength of $0.09 \mathrm{~mm}$.

The results show that although the measured vibration amplitude values are very low a low-frequency vibration is involved in the waviness creation. Indeed, the unbalance created by the rotation of the grinding wheel and dressing disk generates a vibration shaped by the $2 X$ and $1 X$ harmonic of the grinding wheel and dressing disk. Consequently, grinding wheel topography shows a waviness shape at the corresponding beating frequency [Merino 2019].

Without surface topography measurements the diagnosis would not have been possible. The adequate sensors and process variables have been selected and the harmonics of the grinding wheel and dressing disk rotational speeds have been identified thanks to the analysis of both surface topography and vibrations signals.

Other grinding tests with different process conditions ( Tab. 5) confirm the diagnostic. Visual marks observed in a second ground part (test A2) are characterized by a constant distance of $1.54 \mathrm{~mm}$, which corresponds to a frequency of $2.71 \mathrm{~Hz}$ at the given feed rate $(250 \mathrm{~mm} / \mathrm{min})$.

Thus, the beating phenomenon between $2 \mathrm{X}$ and $1 \mathrm{X}$ harmonic of the grinding wheel and dressing disk rotational speeds remains as the main cause of the waviness generated. The proposed approach that combines process and surface topography analysis is essential to find the root cause of the problem.

Tab. 5: Beating tests' process conditions.

\begin{tabular}{|c|c|c|c|c|c|c|c|c|c|c|}
\hline \multirow{3}{*}{ Test } & \multirow{2}{*}{\multicolumn{2}{|c|}{$\begin{array}{c}\text { Grinding } \\
\text { wheel } \\
\text { speed (Ns) }\end{array}$}} & \multirow{2}{*}{\multicolumn{2}{|c|}{$\begin{array}{l}\text { Dressing } \\
\text { speed (ND) }\end{array}$}} & \multirow{3}{*}{$\begin{array}{c}\text { Workpiece } \\
\text { speed }\left(N_{p}\right)\end{array}$} & \multirow{3}{*}{$\begin{array}{c}\text { Dressing } \\
\text { feed rate } \\
\left(V_{f}\right)\end{array}$} & \multicolumn{2}{|c|}{$\begin{array}{l}\text { Measured marks on } \\
\text { the workpiece }\end{array}$} & \multirow{3}{*}{$\begin{array}{c}\begin{array}{c}\text { Beating } \\
\text { frequency }\end{array} \\
2 X N_{s}-1 X N_{D}\end{array}$} & \multirow{3}{*}{$\begin{array}{l}\text { Ra } \\
\mu \mathrm{m}\end{array}$} \\
\hline & & & & & & & \multirow{2}{*}{$\frac{\text { Distance }}{\mathrm{mm}}$} & \multirow{2}{*}{$\frac{\text { Frequency }}{\mathrm{Hz}}$} & & \\
\hline & rpm & $\mathrm{Hz}$ & rpm & $\mathrm{Hz}$ & & & & & & \\
\hline $\mathrm{A} 1$ & 2400 & 40 & 4972 & 82.87 & 160 & 200 & 1.23 & 2.71 & 2.87 & 0.574 \\
\hline $\mathrm{A} 2$ & 2400 & 40 & 4972 & 82.87 & 160 & 250 & 1.54 & 2.71 & 2.87 & 0.487 \\
\hline
\end{tabular}




\section{CONCLUSIONS}

The identification of the root cause of the surface finish problems is critical to solve industrial problems. The proposed methodology that combines surface topography analysis and actual process monitoring eases the diagnostic. Indeed, the detailed analysis of the surface profile allows orienting the diagnostic with the classical monitoring devices in the adequate frequency band. While noise sensors are suitable for high-frequencies, interna signals remain a good alternative for frequencies under 300 $\mathrm{Hz}$. Accelerometers are suitable for a bigger frequency range. Its ease of installation and use makes them a commonly selected alternative for process monitoring. The straightforward relation between cutting forces and the tool tip displacements makes cutting forces monitoring a great alternative. However, the installation of a sensory too holder requires major changes in the machine's structure.

The described methodology to identify machine defects that cause surface topography inaccuracies is corroborated by the identification of beating, unbalance and chatter defects during the machining of rotating workpieces. Unbalance and chatter's vibration frequency are directly related to the profile's wavelength. However, the presence of beating requires a deeper analysis. Indeed, the correlation of surface topography measurements and process monitoring remains essential to find out the defect.

\section{ACKNOWLEDGMENTS}

This research was supported by the InterQ European project (H2020-958357) and is partially funded by the MIRAGED project (CER-20191001 CDTI-CERVERA).

\section{REFERENCES}

[Badger 2011] Badger, J., Murphy, S., \& O'Donnell, G. The effect of wheel eccentricity and run-out on grinding forces, waviness, wheel wear and chatter. International Journal of Machine Tools and Manufacture, 2011, 51(10-11):766-744.

[Bediaga 2013] Bediaga, I., Mendizabal, X., Arnaiz, A., \& Munoa, J. Ball bearing damage detection using traditional signal processing algorithms. IEEE Instrumentation \& Measurement Magazine, 2013, 16(2):20-25.

[Bergs 2019] Bergs, T., Schraknepper, D., \& Goetz, S. Estimation of engagement conditions using an ann pattern recognition system on the base of a sensory tool holder. MM Science Journal, 2019, 1269.

[Brecher 2019] Brecher, C., Eckel, H., Motschke, T., Fey, M., \& Epple, A. Estimation of the virtual workpiece quality by the use of a spindle-integrated process force measurement. CIRP Annals, 2019, 68(1):381-384.

[Cao 2013] Cao, Y., Guan, J., Li, B., Chen, X., Yang, J., \& Gan, C. Modeling and simulation of grinding surface topography considering wheel vibration. The International Journal of Advanced Manufacturing Technology, 2013, 66(5-8):937-945.

[Gao 2019] Gao, W., Haitjema, H., Fang, F., Leach, R. K., Cheung, C. F., Savio, E., \& Linares, J. M. On-machine and in-process surface metrology for precision manufacturing. CIRP Annals, 2019, 68(2):843-866.

[Hockauf 2019] Hockauf, R., Böß, V., Grove, T., \& Denkena, B. Prediction of Ground Surfaces by Using the Actual Tool Topography. Journal of Manufacturing and Materials Processing, 2019, 3(2):40.
[Inasaki 2001] Inasaki, I., Karpuschewski, B. A., \& Lee, H. S. Grinding chatter-origin and suppression. CIRP Annals, 2001, 50(2):515-534.

[Kang 2020] Kang, W., Derani, M., \& Ratnam, M. Effect of Vibration on Surface Roughness in Finish Turning: Simulation Study. Int. J. Simul. Model, 2020, 19:595-606.

[Kapłonek 2015] Kapłonek, W., \& Nadolny, K. Laser methods based on an analysis of scattered light for automated, in-process inspection of machined surfaces: A review. Optik, 2015, 126(20):2764-2770.

[Khare 2015] Khare, S. K., \& Agarwal, S. Predictive modeling of surface roughness in grinding. Procedia CIRP, 2015, 31:375-380.

[Lin, 1998] Lin, S. C., \& Chang, M. F. A study on the effects of vibrations on the surface finish using a surface topography simulation model for turning. International Journal of Machine Tools and Manufacture, 1998, 38(7):763-782.

[Sommer 2019] Sommer, S., Brodmann, B., \& Helfrich, D. Scattering light in bearing production: roundness, waviness, roughness in one operation. In Photonics and Education in Measurement Science, 2019, 11144.

[Stepan 2011] Stepan, G., Dombovari, Z., \& Munoa, J. Identification of cutting force characteristics based on chatter experiments. CIRP annals, 2011, 60(1):113-116.

[Merino 2019] Merino, R., Barrenetxea, D., Munoa, J., \& Dombovari, Z. Analysis of the beating frequencies in dressing and its effect in surface waviness. CIRP Annals, 2019, 68(1):353-356.

[Moehring 2010] Moehring, H. C., Litwinski, K. M., \& Gümmer, O. Process monitoring with sensory machine tool components. CIRP annals, 2010, 59(1):383-386.

[Peng 2004] Peng, Z. K., \& Chu, F. L, Application of the wavelet transform in machine condition monitoring and fault diagnostics: a review with bibliography. Mechanical systems and signal processing, 2004, 18(2):199-221.

[Postel 2019] Postel, M., Aslan, D., Wegener, K., \& Altintas, $Y$. Monitoring of vibrations and cutting forces with spindle mounted vibration sensors. CIRP Annals, 2019, 68(1):413416.

[Randall 2017] Randall, R. B. A history of cepstrum analysis and its application to mechanical problems. Mechanical Systems and Signal Processing, 2017, 97:3-19.

[Ravindra 1997] Ravindra, H., Srinivasa, Y., \& Krishnamurthy, R. Acoustic emission for tool condition monitoring in metal cutting. Wear, 1997, 212(1):78-84.

[Teti 2010] Teti, R., Jemielniak, K., O'Donnell, G., \& Dornfeld, D Advanced monitoring of machining operations. CIRP annals, 2010, 59(2):717-739.

[Whitehouse 1997] Whitehouse, D. J. Surface metrology. Measurement Science and Technology, 1997, 8(9):955.

[Yan 2006] Yan, R., \& Gao, R. X. Hilbert-Huang transformbased vibration signal analysis for machine health monitoring. IEEE Transactions on Instrumentation and measurement, 2016, 55(6):2320-2329.

[Wu, 1996] Wu Y \& Du R. Feature extraction and assessment using wavelet packets for monitoring of machining processes. Mechanical systems and signal processing, 1996, 10(1). 